\title{
What is the recommended size of a Volume of Interest for cancellous bone? A skeleton-based study
}

\author{
G. Dubois ${ }^{a}$, M. Prot ${ }^{\mathrm{a}}$, S. Laporte ${ }^{\mathrm{a}}$ and T.J. Cloete ${ }^{\mathrm{b}}$ \\ a LBM/Institut de Biomécanique Humaine Georges Charpark, Arts et Métiers ParisTech, Paris, France; ${ }^{b}$ Blast Impact and Survivability Research Unit \\ (BISRU), Department of Mechanical Engineering, University of Cape Town (UCT), Rondebosch, South Africa
}

\section{KEYWORDS}

Cancellous bone; size effect; architecture; skeleton

\section{Introduction}

The study of the bone fracture is an important issue for osteoporosis and car safety. The behavior of cancellous bone is strongly linked to the micro-architecture, the strain rate (Prot et al. 2015), and the specimen size (Harrison \& McHugh 2010).

Numerical models are used in order to simulate the viscoelastic behavior up to the point of fracture propagation in cancellous bone. Finite element method (FEM) models based on micro-CT scans are currently the most popular approach. However, the results are dependent on the specimen size and the mesh density, in addition to which the fracture analysis is time-consuming (Hambli 2013). Moreover, the variable architecture within a typical specimen limits the minimum sample size that will still provide reasonable architectural parameter values in comparison with the full specimen size. Indeed, a BV/TV variation up to $20 \%$ was found in the same specimen $(\phi=7.85 \mathrm{~mm}$ ) (Stauber et al. 2014). Skeleton-based models have already shown a great potential for the efficient simulation of bone behavior and fracture. Cancellous bone geometry is based on nodes, beams, and plates (Stauber \& Müller 2006), which is straightforward to implement from a skeleton.

In this study, the effect of the Volume of Interest (VOI) size, within a sample, on the evaluation of cancellous bone architectural parameters from the skeletonized model will be presented. The aim was to furnish recommendations for the sample size for further numerical simulations.

\section{Methods}

\subsection{Specimens}

In total, 126 non-defatted cylindrical cancellous bone specimens $(\phi=10.5 \mathrm{~mm}, H=7.5 \mathrm{~mm}$ ) were extracted from the proximal part of 6 bovine femurs in the three principal planes (sagittal, frontal, and transversal).

\subsection{Architecture acquisition}

A micro-CT scanner (Phoenix, voxel size $80^{3} \mu \mathrm{m}^{3}$ ) was used to acquire the bone architecture. The cancellous bone was automatically segmented from marrow using the Otsu multi-threshold method. Then, the skeleton was thinned from the surface using Avizo software and the following architecture parameters were computed (Table 1).

\subsection{VOl extraction}

From the original specimen models, 11 VOI samples were extracted: 10 were equally radially distributed by maximizing the distance between the VOI centers while one was centered. The vertical positions were randomly generated. The VOI fraction varied from $20 \%$ up to $90 \%$ of the original specimen.

\subsection{Statistical analysis}

For each parameter, the difference between a sample and the original specimen was computed. The mean values of 1386 sample differences (126 specimens $\times 11$ VOIs) were computed for each volume fraction.

\section{Results and discussion}

The influence of the volume fraction on the geometry, morphology, and connectivity is illustrated in Figure 1. A decrease was observed for all parameters with a decrease of the VOI $(p<0.001)$. Tb.Th, Conn.D, and Nd.Nd were the least affected parameters with a mean difference between -5 and $5 \%$. For N.Tp and N.Qp, a mean difference lower than $-5 \%$ was obtained for a minimum volume fraction lower than $40 \%$. This is because the sampling procedure requires the removal of the beams transgressing the VOI boundary. Thus, the number of connections is reduced at the nodes close to the VOI surface. Taking into account the creation of termini during sampling may overcome this bias.

The volume fraction influence on the anisotropy is presented in Figure 2. The value associated with the main direction of the trabeculae, MIL1, was well conserved when the volume fraction 
Table 1. Computed parameters.

\begin{tabular}{lll}
\hline Family & Parameter & Description \\
\hline $\begin{array}{l}\text { Geometry and } \\
\text { morphology }\end{array}$ & $\begin{array}{l}\text { Tb.Th }(\mathrm{mm}) \\
\text { Conn.D }\left(\mathrm{mm}^{-3}\right)\end{array}$ & $\begin{array}{l}\text { Mean thickness of trabeculae } \\
\text { Number of trabeculae per unit } \\
\text { volume }\end{array}$ \\
& Nd.Nd $\left(\mathrm{mm}^{-3}\right)$ & $\begin{array}{l}\text { Average branch length per unit } \\
\text { volume }\end{array}$ \\
Connectivity & N.Tp $\left(\mathrm{mm}^{-3}\right)$ & $\begin{array}{l}\text { Number of triple per unit volume } \\
\text { Number of quadruple per unit } \\
\text { volume }\end{array}$ \\
Anisotropy & N.Qp $\left(\mathrm{mm}^{-3}\right)$ & Mean intercept length \\
\hline
\end{tabular}

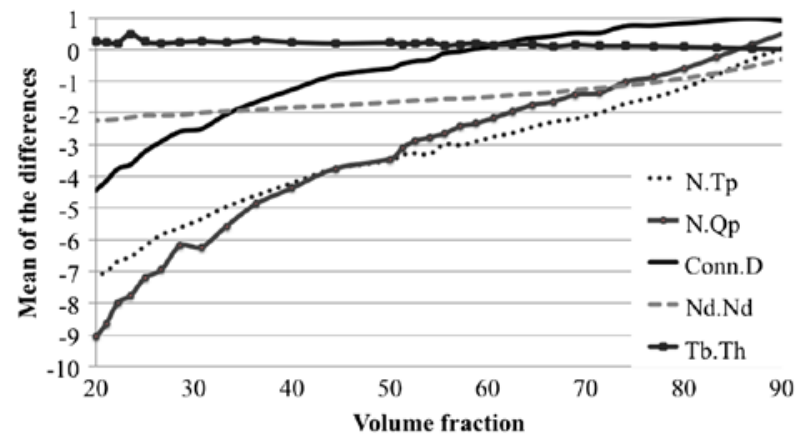

Figure 1. Effect of volume fraction on N.Tp, N.Qp, Conn.D, Nd.Nd, and Tb.Th.

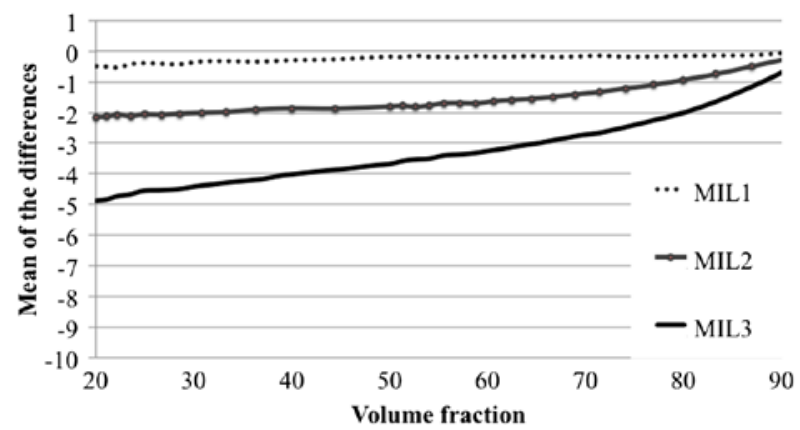

Figure 2. Effect of volume fraction on the mean intercept length.

decreased, with a difference less than $1 \%$. The changes in each MIL are very similar, but they appeared bigger because of the difference in the original MIL values (MIL1 > MIL2 > MIL3).

Harrison and McHugh (2010) have shown a significant decrease of BV/TV when the sample diameter decreased with a constant aspect ratio. In particular, for a volume fraction of $53 \%$, BV/TV decreased to $10 \%$. In a first approximation, a decrease of BV/TV should be reflected in a decrease of Conn.D rather than $\mathrm{Tb}$.Th due its small variation $(<0.5 \%)$. However, Figure 1 shows a decrease of Conn.D of only 1\%. The inho- mogeneity of the limited set of 8 uniaxial specimens used by Harrison and McHugh 2010 may be responsible of the observed difference.

An accurate evaluation of BV/TV is important because it is strongly linked to the apparent Young's modulus $\left(E_{\text {app }}\right)$ as evaluated using FEM simulations (Stauber et al. 2014). Thus, a small variation of BV/TV (and Conn.D) could adversely affect the simulation results. In order to reduce the effect of the volume of the sample on simulations, we assume a maximum mean difference of $\pm 5 \%$ between the VOI and the original specimen. Thus, a volume fraction of $40 \%$ of the original specimen is representative of its skeleton architecture. For cancellous samples from bovine femurs, this implies a minimum representative size of $\phi=7.7 \mathrm{~mm}$ and $H=5.5 \mathrm{~mm}$. This result concurs with a minimum specimen diameter of $7.5 \mathrm{~mm}$ recommended by (Linde et al. 1992) for experiments.

\section{Conclusions}

The VOI sample size has a significant effect on the evaluation of bone architecture parameters. In order to reduce the effect on simulations, the results presented in this study indicate that a sample size of $\phi=7.7 \mathrm{~mm}$ and $H=5.5 \mathrm{~mm}$ is adequate for bovine femoral cancellous bone.

\section{Acknowledgments}

The authors wish to thank Sacks Butcheries for supplying the bovine femurs, Dr. Anton du Plessis from Stellenbosch University for his support with the CT Scanner Facility, Mr. C. Harris (UCT) for the machining of the specimens, and the UCT Research Committee for the financial support to conduct this work.

\section{References}

Hambli R. 2013. Micro-CT finite element model and experimental validation of trabecular bone damage and fracture. Bone. 56:363374.

Harrison NM, McHugh PE. 2010. Comparison of trabecular bone behavior in core and whole bone samples using high-resolution modeling of a vertebral body. Biomech Model Mechanobiol. 9:469480.

Linde F, Hvid I, Madsen F. 1992. The effect of specimen geometry on the mechanical behaviour of trabecular bone specimens. J Biomech. 25:359-368.

Prot M, Saletti D, Pattofatto S, Bousson V, Laporte S. 2015. Links between mechanical behavior of cancellous bone and its microstructural properties under dynamic loading. J Biomech. 48:498-503.

Stauber M, Müller R. 2006. Volumetric spatial decomposition of trabecular bone into rods and plates - a new method for local bone morphometry. Bone. 38:475-484.

Stauber M, Nazarian A, Müller R. 2014. Limitations of global morphometry in predicting trabecular bone failure. J Bone Miner Res. 29:134-141. 